\title{
Transtympanic Route of Administration
}

National Cancer Institute

\section{Source}

National Cancer Institute. Transtympanic Route of Administration. NCI Thesaurus. Code C38309.

Administration of a drug across or through the tympanic cavity. This method reduces the total dose of medication, and allows high concentrations of drug to be delivered directly to the target tissue, while reducing systemic effects. Transtympanic medication can be administered through a needle puncture of the eardrum. If multiple injections are required, a myringotomy tube can be inserted. 